\title{
Fixed-ratio schedules using brain stimulation as a reinforcer ${ }^{1}$
}

SHLOMO I. COHEN ${ }^{2}$ and ROGER W. MCINTIRE, University of Maryland, College Park, Md. 20740

An experiment was conducted to investigate a technique for maintaining behavior on Fixed-Ratio (FR) schedules using one intracranial stimulation (ICS) as a reinforcer. Concurrent schedules programmed on one lever and employing food and ICS rewards were used to establish behavior in Phase 1 to be maintained in Phase 2 by ICS alone. The strength of ICS as a reinforcer in Phase 2 was determined by the combination of food and ICS rewards employed in Phase 1. The results suggest that procedures using small proportions of food reward to ICS reward are useful in establishing behavior to be later maintained by ICS alone after food has been removed.

Much of the work in the literature concerned with intracranial stimulation (ICS) has focused on events physiological in nature. Fewer studies in the ICS literature have been concerned with the use of ICS as a reinforcing stimulus for maintaining operant behavior. A problem was that most investigators considered one ICS of $1 / 2 \mathrm{sec}$ or less duration as approximately equal in reinforcing properties to one pellet, and a generally accepted myth was propagated that there was difficulty in maintaining behavior on intermittent schedules of conventional size with such ICS rewards. Perhaps this myth was responsible for the dearth of investigations focusing on ICS as the reinforcing stimulus. In a review article (Gallistel, 1964), behavior on schedules larger than fixed-ratio (FR) 10 were described as difficult to maintain with ICS reinforcement.

Early studies employed behavior control techniques which were developed when food, not ICS, was used as the reinforcer. These studies used a single isolated event as the reinforcer-one ICS with implied equivalence to one food pellet. Suggestions that ICS was different from conventional reinforcers might have been based on such procedural difficulties and not on difficulties related to the inherent characteristics of ICS. Using ICS to maintain behavior on intermittent schedules required methodological innovations such as the chaining technique described by Pliskoff, Wright, \& Hawkins (1965). In that investigation, reinforcement consisted of opportunity for 20 stimulations rather than only one, and behavior was maintained on schedules of conventional size. In the present experiment, the use of concurrent schedules on a single manipulandum employing food and ICS can be viewed as an additional innovation in developing procedures to maintain behavior of fixed ratio schedules using ICS as a reinforcer.

\section{METHOD}

Three male albino rats of the Wistar strain were implanted with bipolar stainless steel electrodes aimed at the area of the medial forebrain bundle (MFB) posterior to the hypothalamus. At the beginning of the experiment, Ss weighed approximately $300 \mathrm{~g}$ and were maintained between $75 \%$ and $85 \%$ of free feeding weight throughout the experiment.

A Plexiglas chamber with a floor area of $1 \mathrm{sq} f \mathrm{ft}$ was used. One lever approximately $2 \mathrm{in}$. wide protruded $1 \mathrm{in}$. into the chamber and activated a microswitch when pressed with a force of $25 \mathrm{~g}$. The stimulator provided biphasic square wave stimulation with positive and negative excursions of a pulse-pair each $0.2 \mathrm{msec}$ in duration. An "off-time" of $0.2 \mathrm{msec}$ intervened between the two excursions of a pulse-pair. The stimulator delivered 100 pulse-pairs per second.
Stimulation intensities ranged between $500 \mu \mathrm{A}$ to $900 \mu \mathrm{A}$ with a duration of $250 \mathrm{msec}$. White noise was present in the experimental room during all sessions.

A routine surgical preparation as described by Valenstein Hodos, \& Stein (1961) was made. The bregma was located and the electrode was aimed at the MFB area proximal to the mammilary bodies. The coordinates were $5.0 \mathrm{~mm}$ post bregma, $1.0 \mathrm{~mm}$ lateral, and $8.75 \mathrm{~mm}$ vertical. This placement corresponds to AP 3.3, Vertical -3.0, and Lateral 1.0 in the De Groot Atlas (De Groot, 1959). Histological verification of electrode placement was not accomplished for LS 7. The electrode of LS 8 was located at AP 3.3, Vertical -3.3, and Lateral 1.2 (De Groot Atlas). The electrode of LS 11 was located at AP 3.4, Vertical -3.0, and Lateral 1.1. These placements are in the MFB region posterior to the hypothalamus.

After a postsurgery recuperation period of two weeks, all Ss learned to press the lever to receive ICS on continuous reinforcement during a shaping session. Ss were then food deprived and magazine trained.

Phase 1

In Phase 1, ICS and food rewards were programmed on concurrent fixed-ratio schedules with one lever operating on both schedules. The ratio requirement for a food pellet was either equal to or larger than that for ICS. Thus, more than one stimulation per food pellet was delivered except when the ratios were the same. The ratio of the number of ICS rewards to the number of food rewards is called the reward ratio. Concurrent schedules of FR 50 ICS and FR 100 food define a reward ratio of two (ICS) to one (food pellet). Intracranial stimulation rewards were introduced when behavior had stabilized after eight sessions under conditions of FR 50 food.

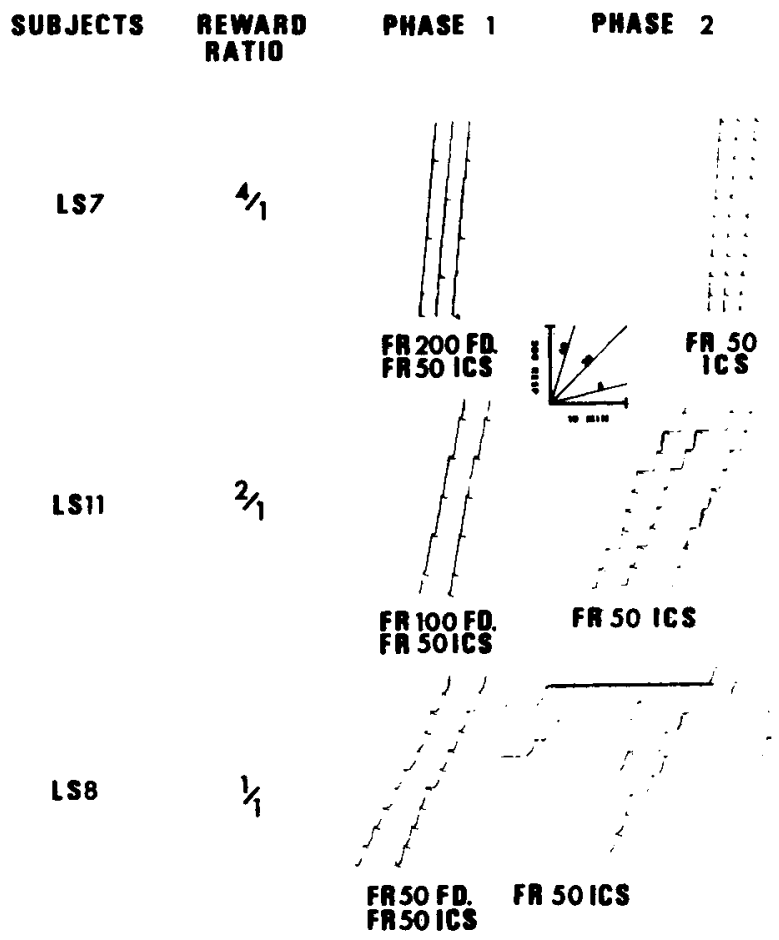

Fig. 1. Characteristic performances of all Ss during Phases 1 and 2. 
Table 1

Mean Post-Reinforcement Pausing in Phase 2

\begin{tabular}{lcc}
\hline Subject & $\begin{array}{c}\text { Reward Ratio (ICS/Food) } \\
\text { in Phase 1 }\end{array}$ & $\begin{array}{c}\text { Mean Pausing } \\
\text { in Phase 2 }\end{array}$ \\
\hline LS 7 & $4 / 1$ & $\begin{array}{c}4.6 \mathrm{sec} \\
\text { LS 11 }\end{array}$ \\
LS 8 & $2 / 1$ & $107.23 \mathrm{sec}$ \\
& $1 / 1$ & $376.00 \mathrm{sec}$ \\
\hline
\end{tabular}

A FR I0 schedule employing ICS reward was then imposed producing concurrent schedules of FR 10 ICS and FR 50 food on the single lever. Following every 10th response ICS was delivered, and following every 50th response ICS and a food pellet were simultaneously delivered. Both ratios of the concurrent schedules were individually increased for each $\mathrm{S}$. Experimental sessions in Phase 1 were defined by the delivery of 40 food pellets or the elapsing of $1 \mathrm{~h}$. The final concurrent schedules defined a unique reward ratio for each $\mathrm{S}$. Thus each $S$ received 40 food pellets per session, while the number of ICS received was a different multiple of 40 .

For LS 7, the final concurrent schedules employed in Phase 1 were FR 50 ICS and FR 200 food (reward ratio $=4 / 1$ ). For LS 11, the final concurrent schedules employed were FR 50 ICS FR 100 food (reward ratio $=2 / 1$ ). For LS 8 , the final concurrent schedules employed were FR 50 ICS FR 50 food (reward ratio $=1 / 1)$.

Phase 2

In Phase 2 of the experiment, food reward was removed from the contingencies leaving ICS alone as the reinforcing stimulus. Phase 2 was initiated after six sessions under conditions of the final concurrent schedules in Phase 1 . Experimental sessions in Phase 2 were uniquely defined for each S. For LS 7, sessions were defined by the delivery of 160 ICS; for LS 11, sessions were defined by the delivery of 80 ICS; for LS 8, sessions were defined by the delivery of 40 ICS. If the appropriate number of ICS reinforcements was not gained, Ss were removed from the experimental chamber after $1 \mathrm{~h}$ elapsed.

\section{RESULTS AND DISCUSSION}

Figure 1 shows characteristic performances of all Ss in Phases 1 and 2 of the present experiment. As the reward ratio during Phase 1 increased, postreinforcement pausing during Phase 2 decreased (see Table 1).

The data suggest that the use of intermittent food reward is helpful in establishing behavior to be later maintained only by intermittent ICS rewards consisting of one stimulation. Such behavior may be difficult to establish when employing a reward of only one ICS. The data further suggest that the different decrements in performance of Ss in Phase 2 may have resulted from a contrast effect due to the removal of food reward.

In Phase 1, the total number of reinforcement events was different for each S. For a reward ratio of $4 / 1$ (LS 7), the total number of reinforeement events consisted of four ICS and one pellet. Food reward comprised $20 \%$ of the total reinforcement events. For a reward ratio of 1/1 (LS 8), food reward comprised $50 \%$ of the total number of reinforcement events. Removal of the food from the Phase 1 contingencies represented removal of a smaller proportion of the reinforcement events as the reward ratio increased. Post ICS reinforcement pauses were shorter in Phase 2 as the food removed represented a smaller proportion of the total number of reinforcing events in Phase 1. Thus, the power of ICS reinforcements to maintain behavior on fixed ratio schedules was inversely related to the proportion of removed reinforcement events represented by the removal of food.

The cumulative records shown in Fig. 1, present stable behavior of Ss after exposure to Phase 2 conditions. During eight sessions of Phase 2, under conditions of FR 50 ICS, LS 7 emitted 64,000 responses at an overall rate greater than two responses per second. S LS 11 emitted 32,000 responses at an overall rate of 67 responses per second. S LS 8 was exposed to Phase 2 for only five experimental sessions and emitted 2473 responses at an overall rate of .10 responses per second.

The suggestion that behavior during Phase 2 was maintained by the conditioned reinforcing properties of ICS is untenable. The posited conditioned reinforcing properties of ICS would have become weaker (perhaps completely extinguished) as ICS was presented during Phase 2 in the absence of food. No evidence of extinction occurred. The large decrease in response rate of LS 8, which occurred when Phase 2 was introduced, did not develop over time, but occurred immediately with the removal of food reward. This supports a contrast hypothesis rather than a conditioned reinforcement hypothesis. These results suggest, therefore, that it is useful to use food and ICS rewards to establish behavior to be maintained later by ICS reward alone. Consideration should be exercised so that the eventual removal of the food reward does not constitute a drastic reduction in the proportion of reinforcing events.

\section{REFERENCES}

De GROOT, J. The rat forebrain in stereotaxic coordinates. Verhandelignen Koninklijke Mederlandse Akademie van Wetenschappen, Naturkunde, $1959,52,1-40$.

GALLISTEL, C. R. Electrical self-stimulation and its theoretical implications. Psychological Bulletin, 1964, 61, 23-34.

PLISKOFF, S. S., WRIGHT, J. E., \& HAWKINS, T. D. Brain stimulation as a reinforcer: Intermittent schedules, Journal of the Analysis of Behavior, 1965, 8, 75-88.

VALENSTEIN, E., HODOS, W., \& STEIN, L. A simplified electrode assembly for implanting chronic electrodes in the brains of: small animals. American Journal of Psychology, 196 1, 74, 125-128. NOTES

1. These data are based on a thesis submitted to the Department of Psychology, University of Maryland, in partial fulfillment of the requirements for the M.A. degree. The research was performed under Contract No. DA49-193-MD-2288 between the Office of the Surgeon General, U.S. Army and the University of Maryland, Stanley S. Pliskoff, Principal Investigator.

2. Now at Kibbutz Gesher Haziv, Mobile Post, Western Galillee, Israel. 